\title{
Some results of common fixed point for four self-maps satisfying a new $\Psi$-contractive condition in partial metric spaces
}

Hui-hui Zheng, Feng Gu*

Institute of Applied Mathematics and Department of Mathematics, Hangzhou Normal University, Hangzhou, Zhejiang 310036, China.

Communicated by Y. Yao

\begin{abstract}
In this paper, we prove some common fixed point theorems for two pairs of weakly compatible selfmaps satisfying a new $\psi$-contractive condition in the framework of a partial metric space. We also provide illustrative examples in support of our new results. The results obtained in this paper differ from the recent relative results in literature. (C)2016 All rights reserved.
\end{abstract}

Keywords: $\psi$-type contractive mapping, common fixed point, coincidence point, partial metric space, weakly compatible mappings.

2010 MSC: 47H10, 54H25.

\section{Introduction and Preliminaries}

In 1994, Matthews [20] introduced the notion of partial metric spaces. In this spaces, the distance of a point to its self may not be zero. In [20], Matthews extended the well known Banach contraction principle from metric spaces to partial metric spaces. Later in [1]-[19] and [21]-[28], several authors obtained some fixed point results for mappings satisfying different contractive conditions.

The purpose of this paper is to use the concept of weakly compatible mappings to discuss some common fixed point problem for four self-maps satisfying a new $\psi$-contractive condition in the framework of a partial metric space. Our results differ from the recent relative results in literature. In fact, as far as now, no author has investigated this problems.

\footnotetext{
* Corresponding author

Email addresses: gufeng_99@163.com (Hui-hui Zheng), zhenghuihui910@sina.com (Feng Gu)
} 
Definition $1.1([20])$. A partial metric on a nonempty set $X$ is a function $p: X \times X \longrightarrow R^{+}$such that for all $x, y, z \in X$ :

$$
\begin{aligned}
& \text { (p1) } p(x, x)=p(x, y)=p(y, y) \Leftrightarrow x=y \\
& (p 2) p(x, x) \leq p(x, y) \\
& \text { (p3) } p(x, y)=p(y, x) \\
& \text { (p4) } p(x, y)+p(z, z) \leq p(x, z)+p(z, y)
\end{aligned}
$$

A partial metric space(PMS for short) is a pair $(X, p)$ such that $X$ is a nonempty set and $\mathrm{p}$ is a partial metric on $X$.

Suppose that $(X, p)$ be a PMS, the function $p^{s}: X \times X \longrightarrow \mathbb{R}^{+}$given by

$$
p^{s}(x, y)=2 p(x, y)-p(x, x)-p(y, y)
$$

is a (usual) metric on $X$. Each partial metric $p$ on $X$ generates a $T_{0}$ topology $\tau_{p}$ on $X$ with a base of the family of open $p$-balls $\left\{B_{p}(x, \varepsilon): x \in X, \varepsilon>0\right\}$, where

$$
B_{p}(x, \varepsilon)=\{y \in X: p(x, y)<p(x, x)+\varepsilon\}
$$

for all $x \in X$ and $\varepsilon>0$.

Definition $1.2([20,23])$. Assume that $(X, p)$ be a PMS,

(1) A sequence $\left\{x_{n}\right\}$ in $(X, p)$ converges to $x \in X$ if and only if $p(x, x)=\lim _{n \rightarrow \infty} p\left(x, x_{n}\right)$.

(2) A sequence $\left\{x_{n}\right\}$ in $(X, p)$ is called a Cauchy if and only if $\lim _{n, m \rightarrow \infty} p\left(x_{n}, x_{m}\right)$ exists (and finite).

(3) A $(X, p)$ is said to be complete if every Cauchy sequence $\left\{x_{n}\right\}$ in $X$ converges, with respect to $\tau_{p}$, to a point $x \in X$ such that $p(x, x)=\lim _{n, m \rightarrow \infty} p\left(x_{n}, x_{m}\right)$.

(4) A mapping $f: X \rightarrow X$ is said to be continuous at $x_{0} \in X$,if for every $\varepsilon>0$, there exists $\delta>0$ such that $f\left(B_{p}\left(x_{0}, \delta\right)\right) \subset B_{p}\left(f\left(x_{0}\right), \varepsilon\right)$.

Example 1.3 ([20]). Suppose that $X=\{[a, b]: a, b \in R, a \leq b\}$ and define $p([a, b],[c, d])=\max \{b, d\}-$ $\min \{a, c\}$. Then $(X, p)$ is a PMS.

Example $1.4([20])$. Assume that $X=[0,+\infty]$ and define $p(x, y)=\max \{x, y\}$, for all $x, y \in X$. Then $(X, p)$ is a complete PMS. It is clear that $p$ is not a (usual) metric.

Lemma 1.5 ([2, 17]). Suppose that $(X, p)$ be a complete PMS. Then

(1) If $p(x, y)=0$ then $x=y$.

(2) If $x \neq y$ then $p(x, y)>0$.

Lemma 1.6 (20, 23]). Assume that $(X, p)$ be a PMS.

(1) The sequence $\left\{x_{n}\right\}$ is Cauchy in $(X, p)$ if and only if $\left\{x_{n}\right\}$ is a Cauchy sequence in $\left(X, p^{s}\right)$.

(2) $(X, p)$ is complete if and only if the metric space $\left(X, p^{s}\right)$ is complete. Moreover,

$$
\lim _{n \rightarrow \infty} p^{s}\left(x, x_{n}\right)=0 \Leftrightarrow p(x, x)=\lim _{n \rightarrow \infty} p\left(x, x_{n}\right)=\lim _{n, m \rightarrow \infty} p\left(x_{n}, x_{m}\right) .
$$

Lemma $1.7\left([23,[2])\right.$. Assume the $x_{n} \rightarrow z$ as $n \rightarrow \infty$ in a PMS $(X, p)$ such that $p(z, z)=0$. Then $\lim _{n \rightarrow \infty} p\left(x_{n}, y\right)=p(z, y)$ for every $y \in X$.

Recall that a pair of self-mappings $\{\mathcal{F}, \mathcal{G}\}$ in a nonempty set $X$ are said to be weakly compatible if

$$
\{t \in X: \mathcal{F} t=\mathcal{G} t\} \subset\{t \in X: \mathcal{F} \mathcal{G} t=\mathcal{G} \mathcal{F} t\} .
$$




\section{Main Results}

In this section, we obtain some unique common fixed point results for four mappings satisfying a new $\psi$-contractive condition in the framework of a partial metric space.

Theorem 2.1. Let $\mathcal{F}, \mathcal{G}, \mathcal{S}$ and $\mathcal{T}$ be four self-maps of a complete $P M S(X, p)$ such that

(i) $\mathcal{F} X \subseteq \mathcal{T} X$ and $\mathcal{G} X \subseteq \mathcal{S} X$;

(ii) one of the ranges $\mathcal{S} X$ and $\mathcal{T} X$ is a closed subset of $(X, p)$;

(iii) the pairs $\{\mathcal{F}, \mathcal{S}\}$ and $\{\mathcal{G}, \mathcal{T}\}$ are weakly compatible and

$$
p^{2}(\mathcal{F} x, \mathcal{G} y) \leq \psi(\mathcal{M}(x, y)), \quad \forall x, y \in X,
$$

where $\psi: \mathbb{R}^{+} \rightarrow \mathbb{R}^{+}$is continuous non-decreasing function such that $\psi(t)<t$ and the series $\Sigma_{n \geq 1}\left[\psi^{n}(t)\right]^{\frac{1}{2}}$ converges for all $t>0$, and

$$
\mathcal{M}(x, y)=\max \left\{\begin{array}{c}
p^{2}(\mathcal{S} x, \mathcal{T} y), p^{2}(\mathcal{F} x, \mathcal{S} x), p^{2}(\mathcal{G} y, \mathcal{T} y), \\
p(\mathcal{S} x, \mathcal{T} y) p(\mathcal{F} x, \mathcal{T} y), p(\mathcal{F} x, \mathcal{S} x) p(\mathcal{F} x, \mathcal{T} y), \\
p(\mathcal{G} y, \mathcal{T} y) p(\mathcal{F} x, \mathcal{T} y), \frac{1}{4}[p(\mathcal{S} x, \mathcal{G} y)+p(\mathcal{F} x, \mathcal{T} y)]^{2}
\end{array}\right\}
$$

for all $x, y \in X$. Then $\mathcal{F}, \mathcal{G}, \mathcal{S}$ and $\mathcal{T}$ have a unique common fixed point in $X$.

Proof. Consider an arbitrary point $x_{0} \in X$. It follows from $\mathcal{F} X \subseteq \mathcal{T} X$ and $\mathcal{G} X \subseteq \mathcal{S} X$ that, there exist two sequences $\left\{x_{n}\right\}$ and $\left\{y_{n}\right\}$ in $X$ satisfying

$$
y_{2 n}=\mathcal{F} x_{2 n}=\mathcal{T} x_{2 n+1} \quad \text { and } \quad y_{2 n+1}=\mathcal{G} x_{2 n+1}=\mathcal{S} x_{2 n+2}
$$

for all $n \in N$.

Next we shall prove that $\left\{y_{n}\right\}$ is a Cauchy sequence in the PMS $(X, p)$.

It follows from $(p 2)$ and $(p 4)$ that

$$
\begin{aligned}
\mathcal{M}\left(x_{2 n}, x_{2 n+1}\right) & =\max \left\{\begin{array}{c}
p^{2}\left(\mathcal{S} x_{2 n}, \mathcal{T} x_{2 n+1}\right), p^{2}\left(\mathcal{F} x_{2 n}, \mathcal{S} x_{2 n}\right), p^{2}\left(\mathcal{G} x_{2 n+1}, \mathcal{T} x_{2 n+1}\right), \\
p\left(\mathcal{S} x_{2 n}, \mathcal{T} x_{2 n+1}\right) p\left(\mathcal{F} x_{2 n}, \mathcal{T} x_{2 n+1}\right), p\left(\mathcal{F} x_{2 n}, \mathcal{S} x_{2 n}\right) p\left(\mathcal{F} x_{2 n}, \mathcal{T} x_{2 n+1}\right), \\
p\left(\mathcal{G} x_{2 n+1}, \mathcal{T} x_{2 n+1}\right) p\left(\mathcal{F} x_{2 n}, \mathcal{T} x_{2 n+1}\right), \\
\left.\frac{1}{4}\left[p\left(\mathcal{S} x_{2 n}, \mathcal{G} x_{2 n+1}\right)+p\left(\mathcal{F} x_{2 n}, \mathcal{T} x_{2 n+1}\right)\right]^{2}\right\}
\end{array}\right\} \\
& =\max \left\{\begin{array}{c}
p^{2}\left(y_{2 n-1}, y_{2 n}\right), p^{2}\left(y_{2 n}, y_{2 n-1}\right), p^{2}\left(y_{2 n+1}, y_{2 n}\right), \\
p\left(y_{2 n-1}, y_{2 n}\right) p\left(y_{2 n}, y_{2 n}\right), p\left(y_{2 n}, y_{2 n-1}\right) p\left(y_{2 n}, y_{2 n}\right), \\
\left.\left.p\left(y_{2 n+1}, y_{2 n}\right) p\left(y_{2 n}, y_{2 n}\right), \frac{1}{4}\left[p\left(y_{2 n-1}, y_{2 n+1}\right)+p\left(y_{2 n}, y_{2 n}\right)\right]^{2}\right\}\right\}
\end{array}\right\} \\
& \leq \max \left\{\begin{array}{c}
p^{2}\left(y_{2 n-1}, y_{2 n}\right), p^{2}\left(y_{2 n}, y_{2 n+1}\right), \\
\left.\frac{1}{4}\left[p\left(y_{2 n-1}, y_{2 n}\right)+p\left(y_{2 n}, y_{2 n+1}\right)\right]^{2}\right\}
\end{array}\right\} \\
& =\max \left\{p^{2}\left(y_{2 n-1}, y_{2 n}\right), p^{2}\left(y_{2 n}, y_{2 n+1}\right)\right\}
\end{aligned}
$$

for all $n \in \mathbb{N}$. By virtue of the property of $\psi$, we get that

$$
\psi\left(\mathcal{M}\left(x_{2 n}, x_{2 n+1}\right)\right) \leq \psi\left(\max \left\{p^{2}\left(y_{2 n-1}, y_{2 n}\right), p^{2}\left(y_{2 n}, y_{2 n+1}\right)\right\}\right) .
$$

By using (2.1) with $x=x_{2 n}, y=y_{2 n+1}$ and (2.3) we obtain

$$
\begin{aligned}
p^{2}\left(y_{2 n}, y_{2 n+1}\right) & =p^{2}\left(\mathcal{F} x_{2 n}, \mathcal{G} x_{2 n+1}\right) \\
& \leq \psi\left(\mathcal{M}\left(x_{2 n}, x_{2 n+1}\right)\right) \\
& \leq \psi\left(\max \left\{p^{2}\left(y_{2 n-1}, y_{2 n}\right), p^{2}\left(y_{2 n}, y_{2 n+1}\right)\right\}\right) .
\end{aligned}
$$

Analogously we can show that

$$
p^{2}\left(y_{2 n+1}, y_{2 n+2}\right) \leq \psi\left(\max \left\{p^{2}\left(y_{2 n}, y_{2 n+1}\right), p^{2}\left(y_{2 n+1}, y_{2 n+2}\right)\right\}\right) .
$$

Note that (2.4) and (2.5) implies that 


$$
p^{2}\left(y_{n}, y_{n+1}\right) \leq \psi\left(\max \left\{p^{2}\left(y_{n-1}, y_{n}\right), p^{2}\left(y_{n}, y_{n+1}\right)\right\}\right) \forall n \geq 1 .
$$

If $\exists n \in \mathbb{N}$ such that $p^{2}\left(y_{2 n-1}, y_{2 n}\right)=0$. Then we have $y_{2 n-1}=y_{2 n}$. It follows from 2.4 that

$$
p^{2}\left(y_{2 n}, y_{2 n+1}\right) \leq \psi\left(p^{2}\left(y_{2 n}, y_{2 n+1}\right)\right) .
$$

Since $\psi(t)<t$ for each $t>0$, with the above inequality we conclude that $p^{2}\left(y_{2 n}, y_{2 n+1}\right)=0$ and so $y_{2 n}=y_{2 n+1}$. Therefore, by 2.5 we get that

$$
p^{2}\left(y_{2 n+1}, y_{2 n+2}\right) \leq \psi\left(p^{2}\left(y_{2 n+1}, y_{2 n+2}\right)\right)
$$

which implies that $y_{2 n+1}=y_{2 n+2}$. Hence, we deduce that $y_{2 n-1}=y_{2 n}=y_{2 n+1}=y_{2 n+2}=\cdots$. Then $\left\{y_{n}\right\}$ is a Cauchy sequence in $(X, p)$. The same conclusion holds if we suppose that there exists $n \in \mathbb{N}$ such that $p^{2}\left(y_{2 n}, y_{2 n+1}\right)=0$ and then $y_{2 n}=y_{2 n+1}$.

Without loss of generality, we may assume that $p^{2}\left(y_{n}, y_{n+1}\right)>0, \forall n \in \mathbb{N}$. Then from 2.5), using the fact that $\psi(t)<t$ for all $t>0$, we have

$$
p^{2}\left(y_{n}, y_{n+1}\right)<\max \left\{p^{2}\left(y_{n-1}, y_{n}\right), p^{2}\left(y_{n}, y_{n+1}\right)\right\} .
$$

Which implies that $p^{2}\left(y_{n}, y_{n+1}\right)<p^{2}\left(y_{n-1}, y_{n}\right)$ and so

$$
\max \left\{p^{2}\left(y_{n-1}, y_{n}\right), p^{2}\left(y_{n}, y_{n+1}\right)\right\}=p^{2}\left(y_{n-1}, y_{n}\right)
$$

Hence, from 2.6 we deduce that

$$
p^{2}\left(y_{n}, y_{n+1}\right) \leq \psi\left(p^{2}\left(y_{n-1}, y_{n}\right)\right) \forall n \geq 1 .
$$

Repeating this inequality $n$ times we get

$$
p^{2}\left(y_{n}, y_{n+1}\right) \leq \psi^{n}\left(p^{2}\left(y_{0}, y_{1}\right)\right) .
$$

It follows from the properties $(p 2)$ and $(p 3)$ that

$$
\max \left\{p^{2}\left(y_{n}, y_{n}\right), p^{2}\left(y_{n+1}, y_{n+1}\right)\right\} \leq p^{2}\left(y_{n}, y_{n+1}\right) .
$$

Hence from 2.8 we have

$$
\max \left\{p^{2}\left(y_{n}, y_{n}\right), p^{2}\left(y_{n+1}, y_{n+1}\right)\right\} \leq \psi^{n}\left(p^{2}\left(y_{0}, y_{1}\right)\right) .
$$

Therefore, from $(p 2),(p 4), 2.8)$ and $(2.9)$ we obtain

$$
\begin{aligned}
{\left[p^{s}\left(y_{n}, y_{n+1}\right)\right]^{2}=} & {\left[2 p\left(y_{n}, y_{n+1}\right)-p\left(y_{n}, y_{n}\right)-p\left(y_{n+1}, y_{n+1}\right)\right]^{2} } \\
\leq & 4 p^{2}\left(y_{n}, y_{n+1}\right)+p^{2}\left(y_{n}, y_{n}\right)+p^{2}\left(y_{n+1}, y_{n+1}\right) \\
& +2 p\left(y_{n}, y_{n}\right) p\left(y_{n+1}, y_{n+1}\right) \\
\leq & 4 p^{2}\left(y_{n}, y_{n+1}\right)+p^{2}\left(y_{n}, y_{n}\right)+p^{2}\left(y_{n+1}, y_{n+1}\right) \\
& +2 \max \left\{p^{2}\left(y_{n}, y_{n}\right), p^{2}\left(y_{n+1}, y_{n+1}\right)\right\} \\
\leq & 8 \psi^{n}\left(p^{2}\left(y_{0}, y_{1}\right)\right) .
\end{aligned}
$$

Hence, we have

$$
p^{s}\left(y_{n}, y_{n+1}\right) \leq\left[8 \psi^{n}\left(p^{2}\left(y_{0}, y_{1}\right)\right)\right]^{\frac{1}{2}} .
$$

Now by the triangle inequality for the metric $p^{s}$ and $(2.10)$, for any $k, n \in \mathbb{N}$, we can get 


$$
\begin{aligned}
p^{s}\left(y_{n}, y_{n+k}\right) & \leq p^{s}\left(y_{n}, y_{n+1}\right)+p^{s}\left(y_{n+1}, y_{n+2}\right)+\cdots+p^{s}\left(y_{n+k-1}, y_{n+k}\right) \\
& \leq\left[8 \psi^{n}\left(p^{2}\left(y_{0}, y_{1}\right)\right)\right]^{\frac{1}{2}}+\left[8 \psi^{n+1}\left(p^{2}\left(y_{0}, y_{1}\right)\right)\right]^{\frac{1}{2}}+\cdots+\left[8 \psi^{n+k-1}\left(p^{2}\left(y_{0}, y_{1}\right)\right)\right]^{\frac{1}{2}} \\
& =2 \sqrt{2} \sum_{i=n}^{n+k-1}\left[\psi^{i}\left(p^{2}\left(y_{0}, y_{1}\right)\right)\right]^{\frac{1}{2}} \\
& \leq 2 \sqrt{2} \sum_{i=n}^{\infty}\left[\psi^{i}\left(p^{2}\left(y_{0}, y_{1}\right)\right)\right]^{\frac{1}{2}} .
\end{aligned}
$$

By virtue of the property of $\psi$ we conclude that for an arbitrary $\varepsilon>0$ there is a positive integer $n_{0}$ satisfying $p^{s}\left(y_{n}, y_{n+k}\right)<\varepsilon$, for every $n \geq n_{0}$ and all $k \in N$. Therefore $\left\{y_{n}\right\}$ is a Cauchy sequence in the metric space $\left(X, p^{s}\right)$.

Since $(X, p)$ is complete PMS, then $\left(X, p^{s}\right)$ is a complete metric space. Therefore, the sequence $\left\{y_{n}\right\}$ converges to some $y \in X$, that is, $\lim _{n \rightarrow \infty} p^{s}\left(y_{n}, y\right)=0$.

Now, we claim that $\lim _{n \rightarrow \infty} p^{2}\left(y_{n}, y\right)=0$. In fact, from Lemma 1.6 (2), we have

$$
p(y, y)=\lim _{n \rightarrow \infty} p\left(y_{n}, y\right)=\lim _{n, m \rightarrow \infty} p\left(y_{n}, y_{m}\right) .
$$

Moreover, since $\left\{y_{n}\right\}$ is a Cauchy sequence in $\left(X, p^{s}\right)$, then $\lim _{n, m \rightarrow \infty} p^{s}\left(y_{n}, y_{m}\right)=0$, and so from (2.8), 2.9. and the property of $\psi$, we have

$$
\lim _{n \rightarrow \infty} p^{2}\left(y_{n}, y_{n}\right)=0 \text { and } \lim _{n \rightarrow \infty} p^{2}\left(y_{n}, y_{n+1}\right)=0 .
$$

Thus from the definition of $p^{s}$ and 2.12 , we have $\lim _{n, m \rightarrow \infty} p\left(y_{n}, y_{m}\right)=0$. So $\lim _{n, m \rightarrow \infty} p^{2}\left(y_{n}, y_{m}\right)=0$. Hence, from (2.11) we have

$$
p^{2}(y, y)=\lim _{n \rightarrow \infty} p^{2}\left(y_{n}, y\right)=\lim _{n, m \rightarrow \infty} p^{2}\left(y_{n}, y_{m}\right)=0 .
$$

This implies that

$$
\lim _{n \rightarrow \infty} p^{2}\left(y_{2 n}, y\right)=\lim _{n \rightarrow \infty} p^{2}\left(y_{2 n+1}, y\right)=0 .
$$

It follows from $(2.2)$ and 2.14 that

$$
\lim _{n \rightarrow \infty} p^{2}\left(\mathcal{F} x_{2 n}, y\right)=\lim _{n \rightarrow \infty} p^{2}\left(\mathcal{T} x_{2 n+1}, y\right)=0
$$

and

$$
\lim _{n \rightarrow \infty} p^{2}\left(\mathcal{G} x_{2 n+1}, y\right)=\lim _{n \rightarrow \infty} p^{2}\left(\mathcal{S} x_{2 n+2}, y\right)=0 .
$$

Assume that $\mathcal{S}(X)$ is a closed subset of the PMS $(X, p)$. From 2.16), there exists $u \in X$ such that $y=\mathcal{S} u$. We claim that $p^{2}(\mathcal{F} u, y)=0$. Otherwise, $p^{2}(\mathcal{F} u, y)>0$. By $(p 2),(p 4)$ and 2.1 we infer that

$$
\begin{aligned}
p^{2}(y, \mathcal{F} u) \leq & {\left[p\left(y, \mathcal{G} x_{2 n+1}\right)+p\left(\mathcal{F} u, \mathcal{G} x_{2 n+1}\right)-p\left(\mathcal{G} x_{2 n+1}, \mathcal{G} x_{2 n+1}\right)\right]^{2} } \\
\leq & p^{2}\left(y, \mathcal{G} x_{2 n+1}\right)+p^{2}\left(\mathcal{F} u, \mathcal{G} x_{2 n+1}\right)+p^{2}\left(\mathcal{G} x_{2 n+1}, \mathcal{G} x_{2 n+1}\right) \\
& +2 p\left(y, \mathcal{G} x_{2 n+1}\right) p\left(\mathcal{F} u, \mathcal{G} x_{2 n+1}\right) \\
\leq & p^{2}\left(y, \mathcal{G} x_{2 n+1}\right)+p^{2}\left(\mathcal{F} u, \mathcal{G} x_{2 n+1}\right)+p^{2}\left(y, \mathcal{G} x_{2 n+1}\right) \\
& +\left[p^{2}\left(y, \mathcal{G} x_{2 n+1}\right)+p^{2}\left(\mathcal{F} u, \mathcal{G} x_{2 n+1}\right)\right] \\
\leq & \left.3 p^{2}\left(y, \mathcal{G} x_{2 n+1}\right)+2 p\left(\mathcal{F} u, \mathcal{G} x_{2 n+1}\right)\right) \\
\leq & 3 p^{2}\left(y, y_{2 n+1}\right)+2 \psi\left(\mathcal{M}\left(u, x_{2 n+1}\right)\right) .
\end{aligned}
$$


On the other hand, it follows from $(2.2),(p 4), 2.13)$ and $(2.14)$ that

$$
\begin{aligned}
& \mathcal{M}\left(u, x_{2 n+1}\right)=\max \left\{\begin{array}{c}
p^{2}\left(\mathcal{S} u, \mathcal{T} x_{2 n+1}\right), p^{2}(\mathcal{F} u, \mathcal{S} u), p^{2}\left(\mathcal{G} x_{2 n+1}, \mathcal{T} x_{2 n+1}\right), \\
p\left(\mathcal{S} u, \mathcal{T} x_{2 n+1}\right) p\left(\mathcal{F} u, \mathcal{T} x_{2 n+1}\right), p(\mathcal{F} u, \mathcal{S} u) p\left(\mathcal{F} u, \mathcal{T} x_{2 n+1}\right), \\
p\left(\mathcal{G} x_{2 n+1}, \mathcal{T} x_{2 n+1}\right) p\left(\mathcal{F} u, \mathcal{T} x_{2 n+1}\right), \\
\frac{1}{4}\left[p\left(\mathcal{S} u, \mathcal{G} x_{2 n+1}\right)+p\left(\mathcal{F} u, \mathcal{T} x_{2 n+1}\right)\right]^{2}
\end{array}\right\} \\
& =\max \left\{\begin{array}{c}
p^{2}\left(y, y_{2 n}\right), p^{2}(\mathcal{F} u, y), p^{2}\left(y_{2 n+1}, y_{2 n}\right), \\
p\left(y, y_{2 n}\right) p\left(\mathcal{F} u, y_{2 n}\right), p(\mathcal{F} u, y) p\left(\mathcal{F} u, y_{2 n}\right), \\
p\left(y_{2 n+1}, y_{2 n}\right) p\left(\mathcal{F} u, y_{2 n}\right), \frac{1}{4}\left[p\left(y, y_{2 n+1}\right)+p\left(\mathcal{F} u, y_{2 n}\right)\right]^{2}
\end{array}\right\} \\
& \leq \max \left\{\begin{array}{c}
p^{2}\left(y, y_{2 n}\right), p^{2}(\mathcal{F} u, y), p^{2}\left(y_{2 n+1}, y_{2 n}\right), \\
p\left(y, y_{2 n}\right)\left[p(\mathcal{F} u, y)+p\left(y, y_{2 n}\right)-p(y, y)\right], \\
p(\mathcal{F} u, y)\left[p(\mathcal{F} u, y)+p\left(y, y_{2 n}\right)-p(y, y)\right], \\
p\left(y_{2 n+1}, y_{2 n}\right)\left[p(\mathcal{F} u, y)+p\left(y, y_{2 n}\right)-p(y, y)\right] \\
\frac{1}{4}\left[p\left(y, y_{2 n+1}\right)+p(\mathcal{F} u, y)+p\left(y, y_{2 n}\right)-p(y, y)\right]^{2}
\end{array}\right\} \\
& =\max \left\{\begin{array}{c}
p^{2}\left(y, y_{2 n}\right), p^{2}(\mathcal{F} u, y), p^{2}\left(y_{2 n+1}, y_{2 n}\right) \\
p\left(y, y_{2 n}\right)\left[p(\mathcal{F} u, y)+p\left(y, y_{2 n}\right)\right] \\
p(\mathcal{F} u, y)\left[p(\mathcal{F} u, y)+p\left(y, y_{2 n}\right)\right] \\
p\left(y_{2 n+1}, y_{2 n}\right)\left[p(\mathcal{F} u, y)+p\left(y, y_{2 n}\right)\right] \\
\frac{1}{4}\left[p\left(y, y_{2 n+1}\right)+p(\mathcal{F} u, y)+p\left(y, y_{2 n}\right)\right]^{2}
\end{array}\right\} .
\end{aligned}
$$

Taking the limit as $n \rightarrow \infty$ in 2.18, we deduce that

$$
\lim _{n \rightarrow \infty} \mathcal{M}\left(u, x_{2 n+1}\right)=p^{2}(\mathcal{F} u, y) .
$$

Since $\psi$ is continuous, from (2.17), 2.19), 2.14), and taking the limit as $n \rightarrow \infty$ we obtain

$$
\begin{aligned}
p^{2}(y, \mathcal{F} u) & \leq \lim _{n \rightarrow \infty}\left[3 p^{2}\left(y, y_{2 n+1}\right)+2 \psi\left(\mathcal{M}\left(u, x_{2 n+1}\right)\right)\right] \\
& =3 \lim _{n \rightarrow \infty} p^{2}\left(y, y_{2 n+1}\right)+2 \psi\left(\lim _{n \rightarrow \infty} \mathcal{M}\left(u, x_{2 n+1}\right)\right) \\
& =2 \psi\left(p^{2}(\mathcal{F} u, y)\right) .
\end{aligned}
$$

Hence, as we supposed that $p^{2}(\mathcal{F} u, y)>0$ and as $\psi(t)<t$ for $t>0$, we have $p^{2}(y, \mathcal{F} u)<2 p^{2}(y, \mathcal{F} u)$, which is impossible. Consequently, $p^{2}(\mathcal{F} u, y)=0$, so that

$$
\mathcal{F} u=y=\mathcal{S} u \text {. }
$$

That is, $u$ is a coincidence point of $\mathcal{F}$ and $\mathcal{S}$.

In view of $y=\mathcal{F} u \in \mathcal{F} X \subseteq \mathcal{T} X$, we deduce that there exists $v \in X$ such that $y=\mathcal{T} v$.

Now we show that $p^{2}(\mathcal{G} v, y)=0$. Otherwise $p^{2}(\mathcal{G} v, y)>0$. Using 2.1 we infer that

$$
p^{2}(y, \mathcal{G} v)=p^{2}(\mathcal{F} u, \mathcal{G} v) \leq \psi(\mathcal{M}(u, v)) .
$$

In light of $y=\mathcal{S} u=\mathcal{F} u=\mathcal{T} v$, we get that

$$
\begin{aligned}
\mathcal{M}(u, v) & =\max \left\{\begin{array}{c}
p^{2}(\mathcal{S} u, \mathcal{T} v), p^{2}(\mathcal{F} u, \mathcal{S} u), p^{2}(\mathcal{G} v, \mathcal{T} v), \\
p(\mathcal{S} u, \mathcal{T} v) p(\mathcal{F} u, \mathcal{T} v), p(\mathcal{F} u, \mathcal{S} u) p(\mathcal{F} u, \mathcal{T} v), \\
p(\mathcal{G} v, \mathcal{T} v) p(\mathcal{F} u, \mathcal{T} v), \frac{1}{4}[p(\mathcal{S} u, \mathcal{G} v)+p(\mathcal{F} u, \mathcal{T} v)]^{2}
\end{array}\right\} \\
& =\max \left\{\begin{array}{c}
p^{2}(y, y), p^{2}(y, y), p^{2}(\mathcal{G} v, y), p^{2}(y, y), \\
\left.p^{2}(y, y), p(\mathcal{G} v, y) p(y, y), \frac{1}{4}[p(y, \mathcal{G} v)+p(y, y)]^{2}\right\}
\end{array}\right\} \\
& =p^{2}(\mathcal{G} v, y) .
\end{aligned}
$$

Making use of (2.21), 2.22) and the property of $\psi$, we deduce that

$$
p^{2}(\mathcal{G} v, y) \leq \psi\left(p^{2}(\mathcal{G} v, y)\right)<p^{2}(\mathcal{G} v, y)
$$

which is a contradiction. Hence $p^{2}(\mathcal{G} v, y)=0$, and so 


$$
\mathcal{G} v=y=\mathcal{T} v .
$$

That is, $v$ is a coincidence point of $\mathcal{G}$ and $\mathcal{T}$.

Since the pair $\{\mathcal{F}, \mathcal{S}\}$ is weakly compatible, it follows from 2.20 that

$$
\mathcal{F} y=\mathcal{F} \mathcal{S} u=\mathcal{S F} u=\mathcal{S} y \text {. }
$$

Now we show that $p^{2}(\mathcal{F} y, y)=0$. We suppose on the contrary that $p^{2}(\mathcal{F} y, y)>0$, we have

$$
\begin{aligned}
p^{2}(\mathcal{F} y, y) \leq & {\left[p\left(\mathcal{F} y, y_{2 n+1}\right)+p\left(y_{2 n+1}, y\right)-p\left(y_{2 n+1}, y_{2 n+1}\right)\right]^{2} } \\
\leq & p^{2}\left(\mathcal{F} y, y_{2 n+1}\right)+p^{2}\left(y_{2 n+1}, y\right)+p^{2}\left(y_{2 n+1}, y_{2 n+1}\right)+2 p\left(\mathcal{A} y, y_{2 n+1}\right) p\left(y_{2 n+1}, y\right) \\
= & p^{2}\left(\mathcal{F} y, \mathcal{G} x_{2 n+1}\right)+p^{2}\left(y_{2 n+1}, y\right)+p^{2}\left(y_{2 n+1}, y_{2 n+1}\right)+2 p\left(\mathcal{F} y, \mathcal{G} x_{2 n+1}\right) p\left(y_{2 n+1}, y\right) \\
\leq & p^{2}\left(\mathcal{F} y, \mathcal{G} x_{2 n+1}\right)+p^{2}\left(y_{2 n+1}, y\right)+p^{2}\left(y_{2 n+1}, y_{2 n+1}\right) \\
& +\left[p^{2}\left(\mathcal{F} y, \mathcal{G}_{2 n+1}\right)+p^{2}\left(y_{2 n+1}, y\right)\right] \\
= & 2 p^{2}\left(\mathcal{F} y, \mathcal{G} x_{2 n+1}\right)+2 p^{2}\left(y_{2 n+1}, y\right)+p^{2}\left(y_{2 n+1}, y_{2 n+1}\right) \\
\leq & 2 \psi\left(\mathcal{M}\left(y, x_{2 n+1}\right)\right)+2 p^{2}\left(y_{2 n+1}, y\right)+p^{2}\left(y_{2 n+1}, y_{2 n+1}\right) .
\end{aligned}
$$

On the other hand, we have

$$
\begin{aligned}
\mathcal{M}\left(y, x_{2 n+1}\right) & =\max \left\{\begin{array}{c}
p^{2}\left(\mathcal{S} y, \mathcal{T} x_{2 n+1}\right), p^{2}(\mathcal{F} y, \mathcal{S} y), p^{2}\left(\mathcal{G} x_{2 n+1}, \mathcal{T} x_{2 n+1}\right), \\
p\left(\mathcal{S} y, \mathcal{T} x_{2 n+1}\right) p\left(\mathcal{F} y, \mathcal{T} x_{2 n+1}\right), p(\mathcal{F} y, \mathcal{S} y) p\left(\mathcal{F} y, \mathcal{T} x_{2 n+1}\right), \\
p\left(\mathcal{G} x_{2 n+1}, \mathcal{T} x_{2 n+1}\right) p\left(\mathcal{F} y, \mathcal{T} x_{2 n+1}\right), \\
\frac{1}{4}\left[p\left(\mathcal{S} y, \mathcal{G} x_{2 n+1}\right)+p\left(\mathcal{F} y, \mathcal{T} x_{2 n+1}\right)\right]^{2}
\end{array}\right\} \\
& =\max \left\{\begin{array}{c}
p^{2}\left(\mathcal{F} y, y_{2 n}\right), p^{2}(\mathcal{F} y, \mathcal{F} y), p^{2}\left(y_{2 n+1}, y_{2 n}\right), p^{2}\left(\mathcal{F} y, y_{2 n}\right), \\
p(\mathcal{F} y, \mathcal{F} y) p\left(\mathcal{F} y, y_{2 n}\right), p\left(y_{2 n+1}, y_{2 n}\right) p\left(\mathcal{F} y, y_{2 n}\right), \\
\frac{1}{4}\left[p\left(\mathcal{F} y, y_{2 n+1}\right)+p\left(\mathcal{F} y, y_{2 n}\right)\right]^{2}
\end{array}\right\}
\end{aligned}
$$

Letting $n \rightarrow \infty$ in the above inequality $(2.25)$, and using Lemma 1.7 and $(2.12)$, we get that

$$
\lim _{n \rightarrow \infty} \mathcal{M}\left(y, x_{2 n+1}\right)=p^{2}(\mathcal{F} y, y) .
$$

Taking the limit as $n \rightarrow \infty$ in (2.24), and in view of (2.26), 2.12), (2.14) and the property of $\psi$, we obtain

$$
p^{2}(\mathcal{F} y, y) \leq 2 \psi\left(p^{2}(\mathcal{F} y, y)\right)<2 p^{2}(\mathcal{F} y, y)
$$

which is a contradiction. Hence $p^{2}(\mathcal{F} y, y)=0$, and so

$$
\mathcal{F} y=y=\mathcal{S} y .
$$

Since the pair $\{\mathcal{G}, \mathcal{T}\}$ is weakly compatible, it follows from 2.23 that

$$
\mathcal{G} y=\mathcal{G} \mathcal{T} v=\mathcal{T G} v=\mathcal{T} y .
$$

We claim that $p^{2}(y, \mathcal{G} y)=0$. Otherwise $p^{2}(y, \mathcal{G} y)>0$. By virtue of 2.1) and (2.27), we obtain

$$
p^{2}(y, \mathcal{G} y)=p^{2}(\mathcal{F} y, \mathcal{G} y) \leq \psi(\mathcal{M}(y, y)) .
$$

On they other hand, in terms of (2.27), $\mathcal{G} y=\mathcal{T} y, 2.13)$ and $(p 2)$, we know that

$$
\mathcal{M}(y, y)=\max \left\{\begin{array}{c}
p^{2}(\mathcal{S} y, \mathcal{T} y), p^{2}(\mathcal{F} y, \mathcal{S} y), p^{2}(\mathcal{G} y, \mathcal{T} y), \\
p(\mathcal{S} y, \mathcal{T} y) p(\mathcal{F} y, \mathcal{T} y), p(\mathcal{F} y, \mathcal{S} y) p(\mathcal{F} y, \mathcal{T} y), \\
p(\mathcal{G} y, \mathcal{T} y) p(\mathcal{F} y, \mathcal{T} y), \frac{1}{4}[p(\mathcal{S} y, \mathcal{G} y)+p(\mathcal{F} y, \mathcal{T} y)]^{2}
\end{array}\right\}
$$




$$
\begin{aligned}
& =\max \left\{\begin{array}{c}
p^{2}(y, \mathcal{G} y), p^{2}(y, y), p^{2}(\mathcal{G} y, \mathcal{G} y), p^{2}(y, \mathcal{G} y), \\
p(y, y) p(y, \mathcal{G} y), p(\mathcal{G} y, \mathcal{G} y) p(y, \mathcal{G} y), \\
\frac{1}{4}[p(y, \mathcal{G} y)+p(y, \mathcal{G} y)]^{2}
\end{array}\right\} \\
& \leq \max \left\{\begin{array}{c}
p^{2}(y, \mathcal{G} y), p^{2}(y, y), p^{2}(y, \mathcal{G} y), p^{2}(y, \mathcal{G} y), \\
p(y, y) p(y, \mathcal{G} y), p(y, \mathcal{G} y) p(y, \mathcal{G} y), \\
\frac{1}{4}[p(y, \mathcal{G} y)+p(y, \mathcal{G} y)]^{2}
\end{array}\right\} \\
& =p^{2}(y, \mathcal{G} y) .
\end{aligned}
$$

Therefore, in view of the property of $\psi$, we infer that

$$
p^{2}(y, \mathcal{G} y)=p^{2}(\mathcal{F} y, \mathcal{G} y) \leq \psi(\mathcal{M}(y, y)) \leq \psi\left(p^{2}(y, \mathcal{G} y)\right)<p^{2}(y, \mathcal{G} y)
$$

This is impossible. Hence $p^{2}(\mathcal{G} y, y)=0$, and so

$$
\mathcal{G} y=y=\mathcal{T} y .
$$

Now, combining (2.27) and (2.28), we obtain

$$
y=\mathcal{F} y=\mathcal{G} y=\mathcal{S} y=\mathcal{T} y .
$$

That is, $y$ is a common fixed point of $\mathcal{F}, \mathcal{G}, \mathcal{S}$ and $\mathcal{T}$.

To prove the uniqueness, suppose that $z$ is another common fixed points of $\mathcal{F}, \mathcal{G}, \mathcal{S}$ and $\mathcal{T}$ and $z \neq y$, then using the contractive condition (2.1), $(p 2)$ and $(p 3)$, we have

$$
\begin{aligned}
p^{2}(y, z) & =p^{2}(\mathcal{A} y, \mathcal{G} z) \\
& \leq \psi\left(\max \left\{\begin{array}{c}
p^{2}(\mathcal{S} y, \mathcal{T} z), p^{2}(\mathcal{F} y, \mathcal{S} y), p^{2}(\mathcal{G} z, \mathcal{T} z), \\
p(\mathcal{S} y, \mathcal{T} z) p(\mathcal{F} y, \mathcal{T} z), p(\mathcal{F} y, \mathcal{S} y) p(\mathcal{F} y, \mathcal{T} z), \\
p(\mathcal{G} z, \mathcal{T} z) p(\mathcal{F} y, \mathcal{T} z), \frac{1}{4}[p(\mathcal{S} y, \mathcal{G} z)+p(\mathcal{F} y, \mathcal{T} z)]^{2}
\end{array}\right\}\right) \\
& =\psi\left(\max \left\{\begin{array}{c}
p^{2}(y, z), p^{2}(y, y), p^{2}(z, z), p^{2}(y, z), \\
p(y, y) p(y, z), p(z, z) p(y, z), p^{2}(y, z)
\end{array}\right\}\right) \\
& \leq \psi\left(\max \left\{\begin{array}{c}
p^{2}(y, z), p^{2}(y, z), p^{2}(y, z), p^{2}(y, z), \\
p(y, z) p(y, z), p(y, z) p(y, z), p^{2}(y, z)
\end{array}\right\}\right) \\
& =\psi\left(p^{2}(y, z)\right) \\
& <p^{2}(y, z) .
\end{aligned}
$$

Which is a contradiction and so must be $z=y$. Consequently, $\mathcal{F}, \mathcal{G}, \mathcal{S}$ and $\mathcal{T}$ have a unique common fixed point.

Assume that $\mathcal{T}(X)$ is a closed subset of the PMS $(X, p)$, then proof is similarly.

This completes the proof.

Remark 2.2. The contractive conditions of Theorem 2.1 is new. As far as now, no author has investigated the problems.

In Theorem 2.1, if $\mathcal{S}=\mathcal{T}$, we deduce the following result of common fixed point for three self-mappings.

Corollary 2.3. Let $\mathcal{F}, \mathcal{G}$ and $\mathcal{S}$ be three self-maps of a complete $P M S(X, p)$ such that

(i) $\mathcal{F} X \subseteq \mathcal{S} X$ and $\mathcal{G} X \subseteq \mathcal{S} X$;

(ii) the ranges $\mathcal{S} X$ is a closed subset of $(X, p)$;

(iii) the pairs $\{\mathcal{F}, \mathcal{S}\}$ and $\{\mathcal{G}, \mathcal{S}\}$ are weakly compatible and

$$
p^{2}(\mathcal{F} x, \mathcal{G} y) \leq \psi(\mathcal{M}(x, y)), \quad \forall x, y \in X,
$$

where $\psi: \mathbb{R}^{+} \rightarrow \mathbb{R}^{+}$is continuous non-decreasing function such that $\psi(t)<t$ and the series 
$\Sigma_{n \geq 1}\left[\psi^{n}(t)\right]^{\frac{1}{2}}$ converges for all $t>0$, and

$$
\mathcal{M}(x, y)=\max \left\{\begin{array}{c}
p^{2}(\mathcal{S} x, \mathcal{S} y), p^{2}(\mathcal{F} x, \mathcal{S} x), p^{2}(\mathcal{G} y, \mathcal{S} y) \\
p(\mathcal{S} x, \mathcal{S} y) p(\mathcal{F} x, \mathcal{S} y), p(\mathcal{F} x, \mathcal{S} x) p(\mathcal{F} x, \mathcal{S} y), \\
p(\mathcal{G} y, \mathcal{S} y) p(\mathcal{F} x, \mathcal{S} y), \frac{1}{4}[p(\mathcal{S} x, \mathcal{G} y)+p(\mathcal{F} x, \mathcal{S} y)]^{2}
\end{array}\right\}
$$

for all $x, y \in X$. Then $\mathcal{F}, \mathcal{G}$ and $\mathcal{S}$ have a unique common fixed point in $X$.

In Theorem 2.1, if $\mathcal{F}=\mathcal{G}$ and $\mathcal{S}=\mathcal{T}$, we deduce the following result of common fixed point for two self-mappings.

Corollary 2.4. $\quad$ Let $\mathcal{F}$ and $S$ be two self-maps of a complete PMS $(X, p)$ such that

(i) $\mathcal{F} X \subseteq \mathcal{S} X$;

(ii) the ranges $\mathcal{S} X$ is a closed subset of $(X, p)$;

(iii) the pairs $\{\mathcal{F}, \mathcal{S}\}$ are weakly compatible and

$$
p^{2}(\mathcal{F} x, \mathcal{F} y) \leq \psi(\mathcal{M}(x, y)), \quad \forall x, y \in X,
$$

where $\psi: \mathbb{R}^{+} \rightarrow \mathbb{R}^{+}$is continuous non-decreasing function such that $\psi(t)<t$ and the series $\Sigma_{n \geq 1}\left[\psi^{n}(t)\right]^{\frac{1}{2}}$ converges for all $t>0$, and

$$
\mathcal{M}(x, y)=\max \left\{\begin{array}{c}
p^{2}(\mathcal{S} x, \mathcal{S} y), p^{2}(\mathcal{F} x, \mathcal{S} x), p^{2}(\mathcal{F} y, \mathcal{S} y) \\
p(\mathcal{S} x, \mathcal{S} y) p(\mathcal{F} x, \mathcal{S} y), p(\mathcal{F} x, \mathcal{S} x) p(\mathcal{F} x, \mathcal{S} y), \\
p(\mathcal{F} y, \mathcal{S} y) p(\mathcal{F} x, \mathcal{S} y), \frac{1}{4}[p(\mathcal{S} x, \mathcal{F} y)+p(\mathcal{F} x, \mathcal{S} y)]^{2}
\end{array}\right\}
$$

for all $x, y \in X$. Then $\mathcal{F}$ and $\mathcal{S}$ have a unique common fixed point in $X$.

In Theorem 2.1, if we take $\mathcal{S}=\mathcal{T}=\mathcal{I}(\mathcal{I}$ is identity mapping, the same below), we deduce the following result of common fixed point for two self-mappings.

Corollary 2.5. Let $\mathcal{F}$ and $\mathcal{G}$ be two self-maps of a complete PMS $(X, p)$ such that

$$
p^{2}(\mathcal{F} x, \mathcal{G} y) \leq \psi(\mathcal{M}(x, y)), \quad \forall x, y \in X,
$$

where $\psi: \mathbb{R}^{+} \rightarrow \mathbb{R}^{+}$is continuous non-decreasing function such that $\psi(t)<t$ and the series $\Sigma_{n \geq 1}\left[\psi^{n}(t)\right]^{\frac{1}{2}}$ converges for all $t>0$, and

$$
\mathcal{M}(x, y)=\max \left\{\begin{array}{c}
p^{2}(x, y), p^{2}(\mathcal{F} x, x), p^{2}(\mathcal{G} y, y) \\
p(x, y) p(\mathcal{F} x, y), p(\mathcal{F} x, x) p(\mathcal{F} x, y), \\
p(\mathcal{G} y, y) p(\mathcal{F} x, y), \frac{1}{4}[p(x, \mathcal{G} y)+p(\mathcal{F} x, y)]^{2}
\end{array}\right\} .
$$

for all $x, y \in X$. Then $\mathcal{F}$ and $\mathcal{G}$ have a unique common fixed point in $X$.

In Theorem 2.1, if $\mathcal{F}=\mathcal{G}$ and $\mathcal{S}=\mathcal{T}=\mathcal{I}$, we deduce the following result of fixed point for one self-mapping.

Corollary 2.6. $\quad$ Let $\mathcal{F}$ be a self-maps of a complete $P M S(X, p)$ such that

$$
p^{2}(\mathcal{F} x, \mathcal{F} y) \leq \psi(\mathcal{M}(x, y)), \quad \forall x, y \in X,
$$

where $\psi: \mathbb{R}^{+} \rightarrow \mathbb{R}^{+}$is continuous non-decreasing function such that $\psi(t)<t$ and the series $\Sigma_{n \geq 1}\left[\psi^{n}(t)\right]^{\frac{1}{2}}$ converges for all $t>0$, and

$$
\mathcal{M}(x, y)=\max \left\{\begin{array}{c}
p^{2}(x, y), p^{2}(\mathcal{F} x, x), p^{2}(\mathcal{F} y, y), \\
p(x, y) p(\mathcal{F} x, y), p(\mathcal{F} x, x) p(\mathcal{F} x, y), \\
p(\mathcal{F} y, y) p(\mathcal{F} x, y), \frac{1}{4}[p(x, \mathcal{F} y)+p(\mathcal{F} x, y)]^{2}
\end{array}\right\}
$$

for all $x, y \in X$. Then $\mathcal{F}$ have a unique fixed point in $X$. 
In Theorem 2.1, if we take $\psi(t)=k t$ and $k \in(0,1)$, then we get the following corollary.

Corollary 2.7. Let $\mathcal{F}, \mathcal{G}, \mathcal{S}$ and $\mathcal{T}$ be four self-maps of a complete PMS $(X, p)$ such that

(i) $\mathcal{F} X \subseteq \mathcal{T} X$ and $\mathcal{G} X \subseteq \mathcal{S} X$;

(ii) one of the ranges $\mathcal{S} X$ and $\mathcal{T} X$ is a closed subset of $(X, p)$;

(ii) the pairs $\{\mathcal{F}, \mathcal{S}\}$ and $\{\mathcal{G}, \mathcal{T}\}$ are weakly compatible and

$$
p^{2}(\mathcal{F} x, \mathcal{G} y) \leq k \max \left\{\begin{array}{c}
p^{2}(\mathcal{S} x, \mathcal{T} y), p^{2}(\mathcal{F} x, \mathcal{S} x), p^{2}(\mathcal{G} y, \mathcal{T} y) \\
p(\mathcal{S} x, \mathcal{T} y) p(\mathcal{F} x, \mathcal{T} y), p(\mathcal{F} x, \mathcal{S} x) p(\mathcal{F} x, \mathcal{T} y) \\
p(\mathcal{G} y, \mathcal{T} y) p(\mathcal{F} x, \mathcal{T} y), \frac{1}{4}[p(\mathcal{S} x, \mathcal{G} y)+p(\mathcal{F} x, \mathcal{T} y)]^{2}
\end{array}\right\}
$$

for all $x, y \in X$, where $k \in(0,1)$ is a constant. Then $\mathcal{F}, \mathcal{G}, \mathcal{S}$ and $\mathcal{T}$ have a unique common fixed point in $X$.

In Corollary 2.7, if $\mathcal{S}=\mathcal{T}$, we deduce the following result of common fixed point for three self-mappings.

Corollary 2.8. Let $\mathcal{F}, \mathcal{G}$ and $\mathcal{S}$ be three self-maps of a complete PMS $(X, p)$ such that

(i) $\mathcal{F} X \subseteq \mathcal{S} X$ and $\mathcal{G} X \subseteq \mathcal{S} X$;

(ii) the ranges $\mathcal{S} X$ is a closed subset of $(X, p)$;

(iii) the pairs $\{\mathcal{F}, \mathcal{S}\}$ and $\{\mathcal{G}, \mathcal{S}\}$ are weakly compatible and

$$
p^{2}(\mathcal{F} x, \mathcal{G} y) \leq k \max \left\{\begin{array}{c}
p^{2}(\mathcal{S} x, \mathcal{S} y), p^{2}(\mathcal{F} x, \mathcal{S} x), p^{2}(\mathcal{G} y, \mathcal{S} y) \\
p(\mathcal{S} x, \mathcal{S} y) p(\mathcal{F} x, \mathcal{S} y), p(\mathcal{F} x, \mathcal{S} x) p(\mathcal{F} x, \mathcal{S} y) \\
p(\mathcal{G} y, \mathcal{S} y) p(\mathcal{F} x, \mathcal{S} y), \frac{1}{4}[p(\mathcal{S} x, \mathcal{G} y)+p(\mathcal{F} x, \mathcal{S} y)]^{2}
\end{array}\right\}
$$

for all $x, y \in X$, where $k \in(0,1)$ is a constant. Then $\mathcal{F}, \mathcal{G}$ and $\mathcal{S}$ have a unique common fixed point in $X$.

In Corollary 2.7, if $\mathcal{F}=\mathcal{G}$ and $\mathcal{S}=\mathcal{T}$, we deduce the following result of common fixed point for two self-mappings.

Corollary 2.9. Let $\mathcal{F}$ and $\mathcal{S}$ be two self-maps of a complete PMS $(X, p)$ such that

(i) $\mathcal{F} X \subseteq \mathcal{S} X$;

(ii) the ranges $\mathcal{S} X$ is a closed subset of $(X, p)$;

(iii) the pairs $\{\mathcal{F}, \mathcal{S}\}$ are weakly compatible and

$$
p^{2}(\mathcal{F} x, \mathcal{F} y) \leq k \max \left\{\begin{array}{c}
p^{2}(\mathcal{S} x, \mathcal{S} y), p^{2}(\mathcal{F} x, \mathcal{S} x), p^{2}(\mathcal{F} y, \mathcal{S} y) \\
p(\mathcal{S} x, \mathcal{S} y) p(\mathcal{F} x, \mathcal{S} y), p(\mathcal{F} x, \mathcal{S} x) p(\mathcal{F} x, \mathcal{S} y), \\
p(\mathcal{F} y, \mathcal{S} y) p(\mathcal{F} x, \mathcal{S} y), \frac{1}{4}[p(\mathcal{S} x, \mathcal{F} y)+p(\mathcal{F} x, \mathcal{S} y)]^{2}
\end{array}\right\}
$$

for all $x, y \in X$, where $k \in(0,1)$ is a constant. Then $\mathcal{F}$ and $\mathcal{S}$ have a unique common fixed point in $X$.

In Corollary 2.7, if $\mathcal{S}=\mathcal{T}=\mathcal{I}$, we deduce the following result of common fixed point for two selfmappings. 
Corollary 2.10. $\quad$ Let $\mathcal{F}$ and $\mathcal{G}$ be two self-maps of a complete $P M S(X, p)$ such that

$$
p^{2}(\mathcal{F} x, \mathcal{G} y) \leq k \max \left\{\begin{array}{c}
p^{2}(x, y), p^{2}(\mathcal{F} x, x), p^{2}(\mathcal{G} y, y) \\
p(x, y) p(\mathcal{F} x, y), p(\mathcal{F} x, x) p(\mathcal{F} x, y), \\
p(\mathcal{G} y, y) p(\mathcal{F} x, y), \frac{1}{4}[p(x, \mathcal{G} y)+p(\mathcal{F} x, y)]^{2}
\end{array}\right\}
$$

for all $x, y \in X$, where $k \in(0,1)$ is a constant. Then $\mathcal{F}$ and $\mathcal{G}$ have a unique common fixed point in $X$.

In Corollary 2.7, if $\mathcal{F}=\mathcal{G}$ and $\mathcal{S}=\mathcal{T}=\mathcal{I}$, we deduce the following result of fixed point for one self-mappings.

Corollary 2.11. Let $\mathcal{F}$ be a self-maps of a complete PMS $(X, p)$ such that

$$
p^{2}(\mathcal{F} x, \mathcal{F} y) \leq k \max \left\{\begin{array}{c}
p^{2}(x, y), p^{2}(\mathcal{F} x, x), p^{2}(\mathcal{F} y, y) \\
p(x, y) p(\mathcal{F} x, y), p(\mathcal{F} x, x) p(\mathcal{F} x, y) \\
p(\mathcal{F} y, y) p(\mathcal{F} x, y), \frac{1}{4}[p(x, \mathcal{F} y)+p(\mathcal{F} x, y)]^{2}
\end{array}\right\}
$$

for all $x, y \in X$, where $k \in(0,1)$ is a constant. Then $\mathcal{F}$ has a unique fixed point in $X$.

Corollary 2.12. Let $\mathcal{F}, \mathcal{G}, \mathcal{S}$ and $\mathcal{T}$ be four self-maps of a complete PMS $(X, p)$ such that

(i) $\mathcal{F} X \subseteq \mathcal{T} X$ and $\mathcal{G} X \subseteq \mathcal{S} X$;

(ii) one of the ranges $\mathcal{S} X$ and $\mathcal{T} X$ is a closed subset of $(X, p)$;

(iii) the pairs $\{\mathcal{F}, \mathcal{S}\}$ and $\{\mathcal{G}, \mathcal{T}\}$ are weakly compatible and

$$
\begin{aligned}
p^{2}(\mathcal{F} x, \mathcal{G} y) \leq & a_{1} p^{2}(\mathcal{S} x, \mathcal{T} y)+a_{2} p^{2}(\mathcal{F} x, \mathcal{S} x)+a_{3} p^{2}(\mathcal{G} y, \mathcal{T} y) \\
& +a_{4} p(\mathcal{S} x, \mathcal{T} y) p(\mathcal{F} x, \mathcal{T} y)+a_{5} p(\mathcal{F} x, \mathcal{S} x) p(\mathcal{F} x, \mathcal{T} y) \\
& +a_{6} p(\mathcal{G} y, \mathcal{T} y) p(\mathcal{F} x, \mathcal{T} y)+a_{7}[p(\mathcal{S} x, \mathcal{G} y)+p(\mathcal{F} x, \mathcal{T} y)]^{2}
\end{aligned}
$$

holds for all $x, y \in X$, where $a_{i} \geq 0(i=1,2,3, \cdots, 7)$ with $a_{1}+a_{2}+a_{3}+a_{4}+a_{5}+a_{6}+4 a_{7}<1$.

Then $\mathcal{F}, \mathcal{G}, \mathcal{S}$ and $\mathcal{T}$ have a unique common fixed point in $X$.

Proof. Let

$$
\mathcal{M}(x, y)=\max \left\{\begin{array}{c}
p^{2}(\mathcal{S} x, \mathcal{T} y), p^{2}(\mathcal{F} x, \mathcal{S} x), p^{2}(\mathcal{G} y, \mathcal{T} y) \\
p(\mathcal{S} x, \mathcal{T} y) p(\mathcal{F} x, \mathcal{T} y), p(\mathcal{F} x, \mathcal{S} x) p(\mathcal{F} x, \mathcal{T} y), \\
p(\mathcal{G} y, \mathcal{T} y) p(\mathcal{F} x, \mathcal{T} y), \frac{1}{4}[p(\mathcal{S} x, \mathcal{G} y)+p(\mathcal{F} x, \mathcal{T} y)]^{2}
\end{array}\right\}
$$

for all $x, y \in X$. Then we have

$$
\begin{aligned}
& a_{1} p^{2}(\mathcal{S} x, \mathcal{T} y)+a_{2} p^{2}(\mathcal{F} x, \mathcal{S} x)+a_{3} p^{2}(\mathcal{G} y, \mathcal{T} y)+a_{4} p(\mathcal{S} x, \mathcal{T} y) p(\mathcal{F} x, \mathcal{T} y) \\
& \quad+a_{5} p(\mathcal{F} x, \mathcal{S} x) p(\mathcal{F} x, \mathcal{T} y)+a_{6} p(\mathcal{G} y, \mathcal{T} y) p(\mathcal{F} x, \mathcal{T} y)+4 a_{7} \cdot \frac{1}{4}[p(\mathcal{S} x, \mathcal{G} y)+p(\mathcal{F} x, \mathcal{T} y)]^{2} \\
& \leq\left(a_{1}+a_{2}+a_{3}+a_{4}+a_{5}+a_{6}+4 a_{7}\right) \mathcal{M}(x, y) .
\end{aligned}
$$

So, if the condition 2.38 hold, then

$$
p^{2}(\mathcal{F} x, \mathcal{G} y) \leq\left(a_{1}+a_{2}+a_{3}+a_{4}+a_{5}+a_{6}+4 a_{7}\right) \mathcal{M}(x, y) .
$$

Taking $k=a_{1}+a_{2}+a_{3}+a_{4}+a_{5}+a_{6}+4 a_{7}$ in Corollary 2.7, the conclusion of Corollary 2.12 can be obtained from Corollary 2.5 immediately.

Remark 2.13. In Corollary 2.12, if we take: (1) $\mathcal{S}=\mathcal{T} ;(2) \mathcal{F}=\mathcal{G} ;(3) \mathcal{F}=\mathcal{G}$ and $\mathcal{S}=\mathcal{T} ;(4) \mathcal{S}=\mathcal{T}=\mathcal{I}$; (5) $\mathcal{F}=\mathcal{G}$ and $\mathcal{S}=\mathcal{T}=\mathcal{I}$, then several new results can be obtained and omit its. 
Now, we give two examples to support Theorem 2.1 .

Example 2.14. Let $X=[0,1]$, and $(X, d)$ be a PMS defined by $p(x, y)=\max \{x, y\}$ for all $x, y \in X$. Let $\mathcal{F}, \mathcal{G}, \mathcal{S}$ and $\mathcal{T}$ be four self mappings defined by

$$
\mathcal{F} x=\frac{x^{2}}{4}, \quad \mathcal{G} x=\frac{x^{2}}{8}, \quad \mathcal{S} x=x^{2}, \quad \mathcal{T} x=\frac{x^{2}}{2}, \quad \forall x \in[0,1] .
$$

Clearly, the subspace $\mathcal{S} X=X$ is closed, $\mathcal{F} X \subset \mathcal{S} X$ and $\mathcal{G} X \subset \mathcal{T} X$. Also, it is easy to show that the pairs $\{\mathcal{F}, \mathcal{S}\}$ and $\{\mathcal{G}, \mathcal{T}\}$ being weakly compatible. In order to check condition 2.1 for all $x, y \in X$ and $\psi(t)=\frac{1}{4} t$ for all $t \in \mathbb{R}^{+}$, we consider the following two cases:

Case 1. If $x \geq y$, then

$$
p^{2}(\mathcal{F} x, \mathcal{G} y)=p^{2}\left(\frac{x^{2}}{4}, \frac{y^{2}}{8}\right)=\frac{x^{4}}{16}
$$

and

$$
p^{2}(\mathcal{F} x, \mathcal{S} x)=p^{2}\left(\frac{x^{2}}{4}, x^{2}\right)=x^{4}
$$

Hence we deduce that

$$
p^{2}(\mathcal{F} x, \mathcal{G} y)=\frac{x^{4}}{16} \leq \frac{x^{4}}{4}=\frac{1}{4} p^{2}(\mathcal{F} x, \mathcal{S} x) \leq \frac{1}{4} \mathcal{M}(x, y)=\psi(\mathcal{M}(x, y)) .
$$

Case 2. If $x<y$, then

$$
p^{2}(\mathcal{F} x, \mathcal{G} y)=p^{2}\left(\frac{x^{2}}{4}, \frac{y^{2}}{8}\right)=\left(\max \left\{\frac{x^{2}}{4}, \frac{y^{2}}{8}\right\}\right)^{2} \leq\left(\max \left\{\frac{y^{2}}{4}, \frac{y^{2}}{8}\right\}\right)^{2}=\frac{y^{4}}{16}
$$

and

$$
p^{2}(\mathcal{G} y, \mathcal{T} y)=p^{2}\left(\frac{y^{2}}{8}, \frac{y^{2}}{2}\right)=\frac{y^{4}}{4}
$$

Therefore we infer that

$$
p^{2}(\mathcal{F} x, \mathcal{G} y) \leq \frac{y^{4}}{16}=\frac{1}{4} p^{2}(\mathcal{G} y, \mathcal{T} y) \leq \frac{1}{4} \mathcal{M}(x, y)=\psi(\mathcal{M}(x, y)) .
$$

Then in all the above cases, the mappings $\mathcal{F}, \mathcal{G}, \mathcal{S}$ and $\mathcal{T}$ are satisfying the condition (2.1) of the Theorem 2.1 with $\psi(t)=\frac{1}{4} t$. So that all the conditions of Theorem 2.1 are fulfilled. Moreover, 0 is the unique common fixed point of $\mathcal{F}, \mathcal{G}, \mathcal{S}$ and $\mathcal{T}$.

Example 2.15. Let $X=\{0,2,3\}$ and let a partial metric $p: X \times X \rightarrow \mathbb{R}^{+}$be defined by $p(x, y)=\max \{x, y\}$ for all $x, y \in X$. Clear, $(X, p)$ is a complete PMS. Let the mappings $\mathcal{F}, \mathcal{G}, \mathcal{S}, \mathcal{T}: X \rightarrow X$ be defined by

Table 1: The definition of maps $\mathcal{F}, \mathcal{G}, \mathcal{S}$ and $\mathcal{T}$ on $X$

\begin{tabular}{lllll}
\hline$x$ & $\mathcal{F}$ & $\mathcal{G}$ & $\mathcal{S}$ & $\mathcal{T}$ \\
\hline 0 & 0 & 0 & 0 & 0 \\
2 & 2 & 0 & 3 & 2 \\
3 & 0 & 2 & 2 & 3 \\
\hline
\end{tabular}

Clearly, the subspace $\mathcal{T} X=X$ is closed, $\mathcal{F} X \subset \mathcal{S} X$ and $\mathcal{G} X \subset \mathcal{T} X$.

Also, it is easy to show that the pairs $\{\mathcal{F}, \mathcal{S}\}$ and $\{\mathcal{G}, \mathcal{T}\}$ being weakly compatible.

In order to verify 2.1 with $\psi(t)=\frac{5}{9} t$ for all $t \in \mathbb{R}^{+}$, we have to consider six possible cases as follows. 
Case (1) $(x, y) \in\{(0,0),(0,2),(3,0),(3,2)\}$, we have $p^{2}(\mathcal{F} x, \mathcal{G} y)=p^{2}(0,0)=0$, and hence 2.1) is obviously satisfied.

Case (2) $(x, y)=(0,3)$, then we have $p^{2}(\mathcal{F} x, \mathcal{G} y)=p^{2}(0,2)=4$ and

$$
\begin{aligned}
\mathcal{M}(0,3) & =\max \left\{\begin{array}{c}
p^{2}(\mathcal{S} 0, \mathcal{T} 3), p^{2}(\mathcal{F} 0, \mathcal{S} 0), p^{2}(\mathcal{G} 3, \mathcal{T} 3) \\
p(\mathcal{S} 0, \mathcal{T} 3) p(\mathcal{F} 0, \mathcal{T} 3), p(\mathcal{F} 0, \mathcal{S} 0) p(\mathcal{F} 0, \mathcal{T} 3), \\
p(\mathcal{G} 3, \mathcal{T} 3) p(\mathcal{F} 0, \mathcal{T} 3), \frac{1}{4}[p(\mathcal{S} 0, \mathcal{G} 3)+p(\mathcal{F} 0, \mathcal{T} 3)]^{2}
\end{array}\right\} \\
& =\max \left\{\begin{array}{l}
\left.9,0,9,9,0,9, \frac{25}{4}\right\} \\
=9 .
\end{array}\right.
\end{aligned}
$$

Thus we conclude that

$$
p^{2}(\mathcal{F} 0, \mathcal{G} 3)=4<5=\frac{5}{9} \cdot 9=\psi(\mathcal{M}(0,3)) .
$$

Case (3) $(x, y)=(2,0)$, then we have $p^{2}(\mathcal{F} x, \mathcal{G} y)=p^{2}(2,0)=4$ and

$$
\begin{aligned}
\mathcal{M}(2,0) & =\max \left\{\begin{array}{c}
p^{2}(\mathcal{S} 2, \mathcal{T} 0), p^{2}(\mathcal{F} 2, \mathcal{S} 2), p^{2}(\mathcal{G} 0, \mathcal{T} 0) \\
p(\mathcal{S} 2, \mathcal{T} 0) p(\mathcal{F} 2, \mathcal{T} 0), p(\mathcal{F} 2, \mathcal{S} 2) p(\mathcal{F} 2, \mathcal{T} 0), \\
p(\mathcal{G} 0, \mathcal{T} 0) p(\mathcal{F} 2, \mathcal{T} 0), \frac{1}{4}[p(\mathcal{S} 2, \mathcal{G} 0)+p(\mathcal{F} 2, \mathcal{T} 0)]^{2}
\end{array}\right\} \\
& =\max \left\{\begin{array}{l}
\left.9,9,0,6,6,0, \frac{25}{4}\right\} \\
=9 .
\end{array}\right.
\end{aligned}
$$

Hence we infer that

$$
p^{2}(\mathcal{F} 2, \mathcal{G} 0)=4<5=\frac{5}{9} \cdot 9=\psi(\mathcal{M}(2,0)) .
$$

Case $(4)(x, y)=(2,2)$, then we have $p^{2}(\mathcal{F} x, \mathcal{G} y)=p^{2}(2,0)=4$ and

$$
\begin{aligned}
\mathcal{M}(2,2) & =\max \left\{\begin{array}{c}
p^{2}(\mathcal{S} 2, \mathcal{T} 2), p^{2}(\mathcal{F} 2, \mathcal{S} 2), p^{2}(\mathcal{G} 2, \mathcal{T} 2) \\
p(\mathcal{S} 2, \mathcal{T} 2) p(\mathcal{F} 2, \mathcal{T} 2), p(\mathcal{F} 2, \mathcal{S} 2) p(\mathcal{F} 2, \mathcal{T} 2), \\
p(\mathcal{G} 2, \mathcal{T} 2) p(\mathcal{F} 2, \mathcal{T} 2), \frac{1}{4}[p(\mathcal{S} 2, \mathcal{G} 2)+p(\mathcal{F} 2, \mathcal{T} 2)]^{2}
\end{array}\right\} \\
& =\max \left\{\begin{array}{l}
\left.9,9,4,6,6,4, \frac{25}{4}\right\} \\
=9 .
\end{array}\right.
\end{aligned}
$$

Therefore we deduce that

$$
p^{2}(\mathcal{F} 2, \mathcal{G} 2)=4<5=\frac{5}{9} \cdot 9=\psi(\mathcal{M}(2,2)) .
$$

Case $(\mathbf{5})(x, y)=(2,3)$, then we have $p^{2}(\mathcal{F} x, \mathcal{G} y)=p^{2}(2,2)=4$ and

$$
\begin{aligned}
\mathcal{M}(2,3) & =\max \left\{\begin{array}{c}
p^{2}(\mathcal{S} 2, \mathcal{T} 3), p^{2}(\mathcal{F} 2, \mathcal{S} 2), p^{2}(\mathcal{G} 3, \mathcal{T} 3) \\
p(\mathcal{S} 2, \mathcal{T} 3) p(\mathcal{F} 2, \mathcal{T} 3), p(\mathcal{F} 2, \mathcal{S} 2) p(\mathcal{F} 2, \mathcal{T} 3), \\
p(\mathcal{G} 3, \mathcal{T} 3) p(\mathcal{F} 2, \mathcal{T} 3), \frac{1}{4}[p(\mathcal{S} 2, \mathcal{G} 3)+p(\mathcal{F} 2, \mathcal{T} 3)]^{2}
\end{array}\right\} \\
& =\max \left\{\begin{array}{l}
9,9,9,9,9,9,9\} \\
=
\end{array}\right.
\end{aligned}
$$

Consequently we know that

$$
p^{2}(\mathcal{F} 2, \mathcal{G} 3)=4<5=\frac{5}{9} \cdot 9=\psi(\mathcal{M}(2,3)) .
$$

Case (6) $(x, y)=(3,3)$, then we have $p^{2}(\mathcal{F} x, \mathcal{G} y)=p^{2}(0,2)=4$ and

$$
\mathcal{M}(3,3)=\max \left\{\begin{array}{c}
p^{2}(\mathcal{S} 3, \mathcal{T} 3), p^{2}(\mathcal{F} 3, \mathcal{S} 3), p^{2}(\mathcal{G} 3, \mathcal{T} 3) \\
p(\mathcal{S} 3, \mathcal{T} 3) p(\mathcal{F} 3, \mathcal{T} 3), p(\mathcal{F} 3, \mathcal{S} 3) p(\mathcal{F} 3, \mathcal{T} 3) \\
p(\mathcal{G} 3, \mathcal{T} 3) p(\mathcal{F} 3, \mathcal{T} 3), \frac{1}{4}[p(\mathcal{S} 3, \mathcal{G} 3)+p(\mathcal{F} 3, \mathcal{T} 3)]^{2}
\end{array}\right\}
$$




$$
\begin{aligned}
& =\max \left\{9,4,9,9,6,9, \frac{25}{4}\right\} \\
& =9 .
\end{aligned}
$$

Hence we get that

$$
p^{2}(\mathcal{F} 3, \mathcal{G} 3)=4<5=\frac{5}{9} \cdot 9=\psi(\mathcal{M}(3,3)) .
$$

Thus, the contractive condition (2.1) is satisfied. And so, all conditions of Theorem 2.1 are satisfied. Moreover, 0 is the unique common fixed point of $\mathcal{F}, \mathcal{G}, \mathcal{S}$ and $\mathcal{T}$.

\section{References}

[1] T. Abdeljawad, Fixed points and generalized weakly contractive mappings in partial metric spaces, Math. Comput. Modelling, 54 (2011), 2923-2927.1

[2] T. Abdeljawad, E. Karapinar, K. Taş, Existence and uniqueness of a common fixed point on partial metric spaces, Appl. Math. Lett., 24 (2011), 1900-1904.1.5 1.7

[3] T. Abdeljawad, E. Karapinar, K. Taş, A generalized contraction principle with control functions on partial metric spaces, Comput. Math. Appl., 63 (2012), 716-719.

[4] M. Akram, E. W. Shamaila, Fixed point results in partial metric spaces using generalized weak contractive conditions, J. Math. Comput. Sci., 12 (2014), 85-98.

[5] I. Altun, Ö Acar, Fixed point theorems for weak contractions in the sense of Berinde on partial metric spaces, Topology Appl., 159 (2012), 2642-2648.

[6] H. Aydi, Some fixed point results in ordered partial metric spaces, J. Nonlinear Sci. Appl., 4 (2011), $210-217$.

[7] H. Aydi, Fixed point theorems for generalized weakly contractive in ordered partial metric spaces, J. Nonlinear Anal. Optim. Theory Appl., 2 (2011), 269-284.

[8] H. Aydi, M. Abbas, C. Vetroc, Partial Hausdorff metric and Nadler's fixed point theorem on partial metric spaces, Topology Appl., 159 (2012), 3234-3242.

[9] C. H. Chen, C. M. Chen, Fixed point results for Meir-Keeler-type $\phi$ - $\alpha$-contractions on partial metric spaces, J. Inequal. Appl., 2013 (2013), 9 pages.

[10] C. Chen, C. Zhu, Fixed point theorems for weakly C-contractive mappings in partial metric spaces, Fixed Point Theory Appl., 2013 (2013), 16 pages.

[11] K. P. Chi, E. Karapinar, T. D. Thanha, A generalized contraction principle in partial metric spaces, Math. Comput. Modelling, 55 (2012), 1673-1681.

[12] L. Ćirić, B. Samet, H. Aydi, C. Vetro, Common fixed point results of generalized contractions on partial metric spaces and application, Appl. Math. Comput., 218 (2011), 2398-2406.

[13] A. Erduran, Z. Kadelburg, H. K. Nashine, C. Vetro, A fixed point theorem for $(\varphi, L)$-weak contraction mappings on a partial metric space, J. Nonlinear Sci. Appl., 7 (2014), 196-204.

[14] D. Ilić, V. Pavlović, V. Rakočević, Some new extensions of Banach's contraction principle to partial metric space, Appl. Math. Lett., 24 (2011), 1326-1330.

[15] D. Ilić, V. Pavlović, V. Rakočević, Extensions of the Zamfirescu theorem to partial metric spaces, Math. Comput. Modelling, 55 (2012), 801-809.

[16] A. Kaewcharoen, T. Yuying, Unique common fixed point theorems on partial metric spaces, J. Nonlinear Sci. Appl., 7 (2014), 90-101.

[17] E. Karapinar, Ćirić types nonunique fixed point theorems on partial metric spaces, J. Nonlinear Sci. Appl., 5 (2012), 74-83.1.5

[18] E. Karapinar, I. Erhan, Fixed point theorems for operators on partial metric spaces, Appl. Math. Lett., 24 (2011), 1894-1899.

[19] E. Karapinar, I. M. Erhan, A. Y. Ulus, Fixed point theorem for cyclic maps on partial metric spaces, Appl. Math. Inf. Sci., 6 (2012), 239-244.1

[20] S. G. Matthews, Partial metric topology. In General Topology and Its Applications, Proc. 8th Summer Conf., Queen's College (1992). Annals New York Acad. Sci., 728 (1994), 183-197.1, 1.1, $1.2,1.3,1.4,1.6$

[21] H. K. Nashine, Z. Kadelburg, S. Radenović, Common fixed point theorems for weakly isotone increasing mappings in ordered partial metric spaces, Math. Comput. Modelling, 57 (2013), 2355-2365.11

[22] T. Nazir, M. Abbas, Common fixed points of two pairs of mappings satisfying (E.A)-property in partial metric spaces, J. Inequal. Appl., 2014 (2014), 12 pages.

[23] S. Oltra, O. Valero, Banach's fixed point theorem for partial metric spaces, Rend. Ist. Mat. Univ. Trieste, 36 (2004), 17-26.1.2 $1.6,1.7$

[24] V. L. Rosa, P. Vetro, Fixed points for Geraghty-contractions in partial metric spaces, J. Nonlinear Sci. Appl., 7 (2014), 1-10.

[25] W. Shatanawi, H. K. Nashine, A generalization of Banach's contraction principle for nonlinear contraction in a partial metric space, J. Nonlinear Sci. Appl., 5 (2012), 37-43. 
[26] W. Shatanawi, M. Postolache, Coincidence and fixed point results for generalized weak contractions in the sense of Berinde on partial metric spaces, Fixed Point Theory Appl., 2013 (2013), 17 pages.

[27] F. Vetro, S. Radenovic, Nonlinear w-quasi-contractions of Óirić-type in partial metric spaces, Appl. Math. Comput., 219 (2012), 1594-1600.

[28] C. Vetro, F. Vetro, Common fixed points of mappings satisfying implicit relations in partial metric spaces, J. Nonlinear Sci. Appl., 6 (2013), 152-161.1 